\title{
Phenotypic Properties of Collagen in Dentinogenesis Imperfecta Associated with Osteogenesis Imperfecta
}

This article was published in the following Dove Press journal:

International Journal of Nanomedicine

\author{
Salwa Ibrahim ${ }^{l} * *$ \\ Adam P Strange ${ }^{2, *}$ \\ Sebastian Aguayo (D) ${ }^{2,3}$ \\ Albatool Shinawi ${ }^{1}$ \\ Nabilah Harith ' \\ Nurjehan Mohamed- \\ Ibrahim ' \\ Samera Siddiqui ${ }^{2}$ \\ Susan Parekh ${ }^{1}$ \\ Laurent Bozec ${ }^{4}$ \\ 'Department of Paediatric Dentistry, \\ UCL Eastman Dental Institute, University \\ College London, London, UK; \\ ${ }^{2}$ Department of Biomaterials and Tissue \\ Engineering, UCL Eastman Dental \\ Institute, University College London, \\ London, UK; ${ }^{3}$ School of Dentistry, \\ Faculty of Medicine, Pontificia \\ Universidad Católica de Chile, Santiago, \\ Chile; ${ }^{4}$ Faculty of Dentistry, University of \\ Toronto, Toronto, Canada \\ *These authors contributed equally to \\ this work
}

Introduction: Dentinogenesis imperfecta type 1 (OIDI) is considered a relatively rare genetic disorder (1:5000 to 1:45,000) associated with osteogenesis imperfecta. OIDI impacts the formation of collagen fibrils in dentin, leading to morphological and structural changes that affect the strength and appearance of teeth. However, there is still a lack of understanding regarding the nanoscale characterization of the disease, in terms of collagen ultrastructure and mechanical properties. Therefore, this research presents a qualitative and quantitative report into the phenotype and characterization of OIDI in dentin, by using a combination of imaging, nanomechanical approaches. Methods: For this study, 8 primary molars from OIDI patients and 8 primary control molars were collected, embedded in acrylic resin and cut into longitudinal sections. Sections were then demineralized in $37 \%$ phosphoric acid using a protocol developed in-house. Initial experiments demonstrated the effectiveness of the demineralization protocol, as the ATRFTIR spectral fingerprints showed an increase in the amide bands together with a decrease in phosphate content. Structural and mechanical analyses were performed directly on both the mineralized and demineralized samples using a combination of scanning electron microscopy, atomic force microscopy, and Wallace indentation.

Results: Mesoscale imaging showed alterations in dentinal tubule morphology in OIDI patients, with a reduced number of tubules and a decreased tubule diameter compared to healthy controls. Nanoscale collagen ultrastructure presented a similar D-banding periodicity between OIDI and controls. Reduced collagen fibrils diameter was also recorded for the OIDI group. The hardness of the (mineralized) control dentin was found to be significantly higher $(p<0.05)$ than that of the OIDI (mineralized) dentine. Both the exposed peri- and intratubular dentinal collagen presented bimodal elastic behaviors (Young's moduli). The control samples presented a stiffening of the intratubular collagen when compared to the peritubular collagen. In case of the OIDI, this stiffening in the collagen between peri- and intratubular dentinal collagen was not observed and the exposed collagen presented overall a lower elasticity than the control samples.

Conclusion: This study presents a systematic approach to the characterization of collagen structure and properties in OIDI as diagnosed in dentin. Structural markers for OIDI at the mesoscale and nanoscale were found and correlated with an observed lack of increased elastic moduli of the collagen fibrils in the intratubular OIDI dentin. These findings offer an explanation of how structural changes in the dentin could be responsible for the failure of some adhesive restorative materials as observed in patients affected by OIDI.

Keywords: dentin, collagen, dentinogenesis imperfecta, demineralisation, dentistry, atomic force microscopy

\section{Introduction}

Dentinogenesis imperfecta (DI), or opalescent teeth disease, is an inherited disorder in which dentin quality is affected. DI is a localized mesodermal dysplasia affecting
Correspondence: Laurent Bozec Faculty of Dentistry, University of Toronto, 124 Edward Street, Toronto ON M5G IG6, Canada

Email L.bozec@utoronto.ca 
both primary and permanent dentition. The first classification of DI was originally proposed by Shields ${ }^{1}$ in 1973 and was further commented by Wiktop ${ }^{2}$ in 1975. A more recent report $^{3}$ reviewed the evolution in the etiology and nomenclature system of this condition to clarify the relationship between DI and osteogenesis imperfecta (OI). According to Shields, ${ }^{1}$ DI is classified into three main groups, Type I (DI associated with OI (osteogenesis imperfecta), an autosomal dominant trait), Type II (also an autosomal dominant trait, but with no OI association), and Type III (thought to have occurred as variable of DI Type II). The differential diagnosis between types I and II is often challenging, possibly due to the rarity of the conditions. The prevalence of DI was recently found to be as few as 1 in 45,000 in Swedish children and adolescents, $^{4}$ although ORPHANET (ORPHA:71267) cites the prevalence of DI to be $<1 / 1,000,000$. DI type III, also called "Brandywine isolate", is described as an extremely rare form with highest incidence of 1 in 15 in the sub-population of Maryland. ${ }^{5}$

Clinical and radiographic representation of DI varies greatly between, and within types. In DI type I (also denoted in this manuscript as OIDI), primary predecessors are usually more severely affected than permanent teeth. In DI type II, both dentitions are equally affected. Radiographically, DI type II and primary dentition in DI type I, show pulp obliteration, unlike type III which is characterized by thin dentin and large pulp chambers. DI type III teeth are frequently referred to as "Shell Teeth". 6 Furthermore, DI affected teeth will often have short roots and bulbous crowns, with a significant constriction at cement-enamel junction. ${ }^{7}$ Intraorally, teeth are described as being translucent with a discolouration hue ranging from yellowish brown to greyish blue, and an increased susceptibility to wear and fracture. It is believed that the cause of frequent tooth wears and enamel breakage may be due to defective EDJ (Enamel Dentin Junction). ${ }^{8}$

The primary cause of the autosomal dominant DI type I (OIDI) is mutations in COL1A1 or COL1A2 genes. These genes encode for the $\alpha-1$ and $\alpha-2$ polypeptide chains of collagen type I. ${ }^{9-12}$ These polypeptides chains are the basic building blocks of the collagen molecules, and therefore any alterations in the structural integrity of the molecules may trigger abnormal collagen fibril formation. Genetic mutations causing OIDI are often linked to the substitution of the glycine (Gly) residue present in the $\alpha-1$ and/or $\alpha-2$ polypeptide chains by other larger residues such as serine. ${ }^{13}$ Being the smallest amino acid within the $\alpha-1$ and $\alpha-2$ polypeptide chains, Gly often sits within the centre of the triple helical structure of collagen, allowing the helices to wind together tightly. The Gly residue is also involved in the stabilization of the triple helix through the formation of hydrogen bonds with the internal bound water. ${ }^{14}$ Thus, any changes in identity of the amino acid replacing Gly, and the location of that substitution, will alter the ability of the collagen triple helix to form correctly, and impact the pathology of osteogenesis imperfecta (OI). For example, substitutions of glycine with serine (polar amino acid) in the $\alpha-1$ collagen chains have been found to cause severe types of OI in bones. ${ }^{15}$ Similarly, it has been established that patients with glycine substitutions will have some degree of OIDI comorbidity. ${ }^{16-19}$

While the effect of OI on bones has been well reported and studied, there is a lack of reported outcomes for OIDI in teeth. In this study, we present recent findings in the structure and properties of OIDI-affected dentin and especially in its collagen component. Using a combination of imaging and mechanical approaches, we present a quantitative and qualitative report of the phenotype of DI associated with OI in teeth.

\section{Materials and Methods Tooth Collection and Preparation}

Ethical approval for this study was obtained from the National Health Services Research Ethics Committee (registration reference number 11/LO/0777). Following written informed consent, 8 primary first and second molars from patients with a confirmed genetic diagnosis of OI (OIDI), and 8 primary first and second molars from healthy control individuals were collected over a period of 24 months, at the University College London Eastman Dental Hospital, London, UK. All patients with OI underwent genetic testing at Great Ormond Street Hospital (London UK). Those included in this study had all a confirmed diagnosis of mutations in COLA1 gene and had not been treated with bisphosphonate therapy. Figure 1 shows the vertical bitewing radiographs of one of the patients with OI Type I and OIDI in the primary dentition, showing characteristic features of pulpal obliteration and bulbous crowns. All teeth were extracted as part of the patient's treatment plan. It is worth noting that due to the rarity of the condition, accessing OIDI teeth is one of the major limitations in any study of this disease, and as a result, this study could not be powered.

Following extraction, the teeth were initially stored in a $70 \%$ ethanol solution for up to 5 days at room temperature, before being debrided from the remaining soft tissues, and finally stored in a $0.1 \%$ thymol solution at $4^{\circ} \mathrm{C}$ until required 


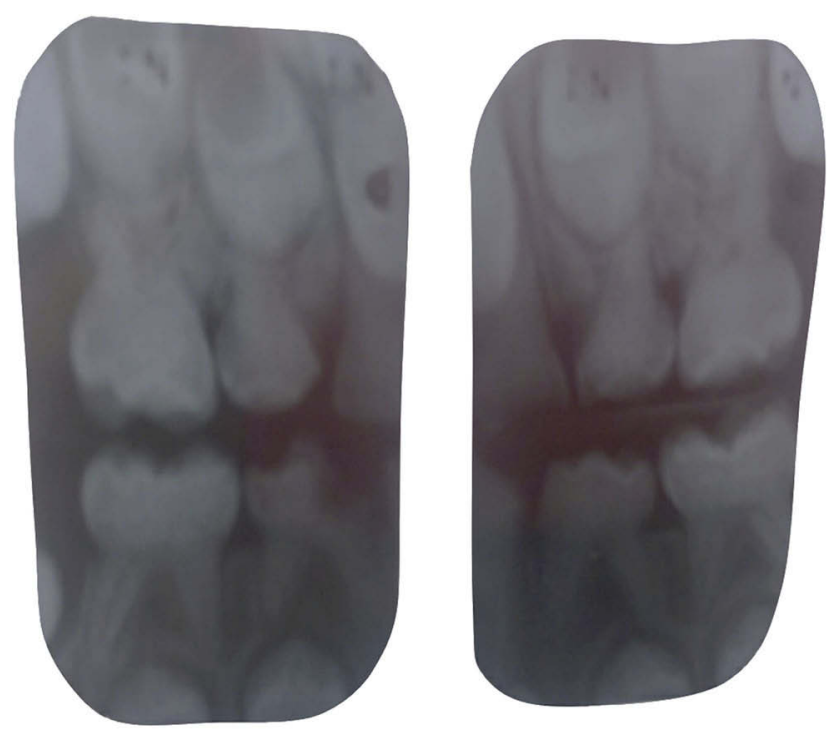

Figure I Vertical bitewing radiographs of patient with OIDI in the primary dentition, showing characteristic features of pulpal obliteration and bulbous crowns.

for the study (storage did not exceed 2 months). The teeth were then sectioned longitudinally ( $\sim 1-1.5 \mathrm{~mm}$ thick) using a diamond microtome (Struers, Accutom-50, Struers Ltd., Solihull, West Midlands, UK). Finally, the sections were then sonicated in Ultra-High Quality (UHQ) water for $30 \mathrm{~s}$.

\section{Dentin Hardness}

To evaluate the hardness of the dentin, the sections were then mounted in a Wallace indenter (H.W. Wallace, Croydon, England). The sections were held in position on the sample platform using a malleable putty to prevent any sliding of the sections during the measurements. By measuring the indentation depth of the indenter ( $40 \mu \mathrm{m}$ pyramidal shape), one can obtain the Vickers Hardness Number for the samples using the following equation:

$$
V H N=\frac{\text { applied force }(F)}{\text { surface area }}=1854.4 \times \frac{F}{d^{2}}
$$

where $\mathrm{F}$ is applied load (300 $\mathrm{g})$ and $\mathrm{d}$ is the mean diagonal of indentation (in $\mu \mathrm{m}$ ). All measurements were performed by maintaining the load for $15 \mathrm{~s}$ before release. For each tooth, a total of 10 individual measurements were taken within the crown region of each section. The VHN values were plotted as boxplots focussing on the two groups, control vs OIDI.

\section{Controlled Exposure of the Collagen Network}

Following hardness measurements, sections were then demineralized in a solution of $37 \%$ phosphoric acid placed in an ultrasonic bath. The exposure time of the section to the acid was closely monitored by Attenuated Total ReflectanceFourier Transform Infrared Spectroscopy (ATR-FTIR) using a Tensor 27 spectrometer (Bruker, Coventry, UK) equipped with a GladiATR (PIKE Technologies, Madison, USA). Following time-controlled acid-exposure, the sections were then washed with UHQ water, before being placed in $6.5 \%$ sodium hypochlorite (ReAgent, Liverpool, UK) for 5 $\mathrm{s}$ to remove any smear layer resulting from the acid etching process. Finally, the sections were washed again in UHQ water before being air-dried for $24 \mathrm{hrs}$ prior to any measurements. This approach was successfully applied to other studies. ${ }^{17}$ All infrared spectra were recorded at 128 coadditions and $16 \mathrm{~cm}^{-1}$ spectral resolution over the spectral range 4000-700 $\mathrm{cm}^{-1}$. The spectra were baseline corrected before being normalized using the Amide I band at $1650 \mathrm{~cm}^{-1}$. The potency of the demineralization protocol was assessed by calculating the normalised ratio of spectral intensities of the phosphate band over the Amide I band.

\section{Imaging the Collagen Network (by AFM)}

No further sample preparation was required for atomic force microscopy (AFM) imaging. Individual sections were mounted onto glass-slides using double-sided tape and kept in ambient conditions. AFM imaging was performed using a Dimension 3100 Atomic Force Microscope (Bruker, Santa Barbara, CA, USA). Imaging was carried out with an MNSL AFM probe $(\mathrm{k}=0.03-0.1 \mathrm{~N} / \mathrm{m})$ (Bruker, Santa Barbara, CA, USA) in contact mode in air. Set point and gain values were adjusted in-situ during scanning for image optimization. Images were obtained at $512 \times 512$ pixels (image size: $10 \times 10 \mu \mathrm{m}^{2}$ or $2 \times 2 \mu \mathrm{m}^{2}$ ) with an average scanning rate of $1 \mathrm{~Hz}$. Images were obtained and analysed using Nanoscope Analysis Software (Version v14, Bruker, Santa Barbara, CA, USA) in order to measure both fibrils diameter (cross-section profile analysis) and D-banding periodicity (line-profile analysis along the fibrils length).

\section{Single Fibrils Collagen Nanomechanics (by AFM)}

For the mechanical measurements carried out on the exposed collagen matrix, a Nanowizard AFM (JPK, Berlin, Germany) mounted on an inverted optical microscope (Olympus IX71, Olympus, Japan) was used. Mechanical measurements were performed using REFSPA tips $(\mathrm{k}=3 \mathrm{~N} / \mathrm{m}$ ) (Bruker, Santa Barbara, CA, USA) in ambient conditions (prior to the SEM sample processing). For all measurements, both the 
indentation load $(5 \mathrm{nN})$ and loading rate were kept constant. Prior to acquiring any force-distance curves (FD), a lowresolution image $\left(256 \times 256\right.$ pixels $\left.-5 \times 5 \mu^{2}\right)$ was acquired in contact mode with the same probe to ensure that the area being characterized mechanically presented sufficient exposed collagen matrix. FD measurements were then conducted on individual collagen fibrils presenting a D-banding periodicity by choosing the centre of the D-banding (overlap region) as indentation site on the low-resolution AFM image in both the intra-tubular and peri-tubular dentin. At least three series of indentation curves were taken in each location (7) for each sample, with at least three sections per condition analysed, for a minimal of 350 force curves per location. FD curves were analysed on proprietary software (JPK, Germany) and the Young's modulus was calculated using the Sneddon model. $^{20}$ All force curves were analysed using the JPK Data Processing Software v.5.1.8 (JPK Instruments, Germany).

\section{Imaging the Collagen Network (by SEM)}

Following AFM characterization, the sections were fixed for $24 \mathrm{hrs}$ in 3\% glutaraldehyde (Agar Scientific, UK) and were then dehydrated using ethanol series, before being thinly coated with gold-palladium using a Polaron E5000 Sputter Coater (Quorum Technologies Ltd, East Sussex, UK). Scanning Electron Microscopy (SEM) imaging was performed using an FEI XL30 FEG-SEM (FEI, Eindhoven, Netherlands) using a $5 \mathrm{kV}$ accelerating voltage. A total of 7 locations were selected at random for each section and imaged at different magnifications.

\section{Statistical Analyses}

One-way ANOVA (Kruskal-Wallis test) was conducted to assess the significance $(\mathrm{p}<0.05)$ for the mechanical and structural parameters presented as boxplots (median and 95\% CI) between the control and OIDI groups. For the nanomechanics analyses, histograms and median values were obtained for the Young's moduli. All data were plotted and processed using Origin software (OriginLab Corp, Northampton, MA, USA).

\section{Results and Discussion}

\section{Systematic Demineralization of Dentin}

\section{Surface}

Dentin is the mineralized matrix situated within the core of the tooth. Structurally, it can be characterized by multiple tightly packed dentinal tubules found along its entire thickness. After dentinogenesis is completed, $70 \%$ by weight of dentin is mineralized hydroxyapatite, with the organic content accounting for $20 \%$ of the matrix, and the remaining $10 \%$ being water. Fibrillar collagen makes up the organic component of dentin, $85 \%$ type I collagen, and $15 \%$ types III and V collagen. To date, efforts in demineralising dentin surfaces to expose collagen have had mitigated success, in terms of repeatability. This can be explained by the fact that dentin is a heterogeneous tissue, and as such, it reacts differently when exposed to demineralisation agents. However, to access the ultrastructure of the collagen scaffold present within such a mineralized matrix, it is essential to demineralize this matrix, but without altering the morphology and chemistry of the collagen. To control the degree of demineralisation of the dentin surface, and to maintain the clinical relevance of our approach, we decided to use $37 \%$ phosphoric acid as it is commonly used in most clinical dental etching protocols. Other acids such as citric, lactic or nitric acids, and chelation solutions such as EDTA, have all been used to remove minerals from bone or dentin samples. Unfortunately, collagen crosslinks are very susceptible to acid attack and will be cleaved when exposed to a sustained acidic environment. Although chelation agents would not affect these crosslinks, their demineralization action is a slow process and is therefore not clinically relevant.

Figure 2A shows the normalized FTIR data for control (dotted line) and demineralized dentin (full line) after 10 s exposure to phosphoric acid. In the FTIR spectrum of the native control dentin, the presence of mineral phase of the dentin, hydroxyapatite (HA), dominates the spectral fingerprint. The most characteristic chemical groups in the FTIR spectrum of $\mathrm{HA}$ are $\mathrm{PO}_{4}{ }^{3-}, \mathrm{OH}^{-}$and $\mathrm{CO}_{3}{ }^{2-}$. $\mathrm{PO}_{4}{ }^{3-}$ group presents intensive IR absorption bands at 560 and $600 \mathrm{~cm}^{-1}$ (not shown) and at $1000-1100 \mathrm{~cm}^{-1}$. The Amide A band is relatively wide, from 3600 to $2600 \mathrm{~cm}^{-1}$, with an explicit peak at $3570 \mathrm{~cm}^{-1} \cdot \mathrm{CO}_{3}{ }^{2-}$ group forms weak peaks between 870 and $880 \mathrm{~cm}^{-1}$ and more intensive peaks between 1460 and $1530 \mathrm{~cm}^{-1}$. In contrast, in the FTIR spectrum of the demineralized control dentin, the presence of protein phase of the dentin, collagen, dominates the spectral fingerprint. Collagen infrared fingerprint is defined by several specific absorption bands defined as Amide A, I, II and III. The Amide A band is the broad peak present in the spectrum between 3600 and $3200 \mathrm{~cm}^{-1}$ which relates to $\mathrm{N}-$ $\mathrm{H}$ stretching vibrations. The amide bonds present in the collagen amino-structure contributes significantly to the FTIR spectrum through both stretching and bending vibrations of the carbonyl $(\mathrm{C}=\mathrm{O})$ at $1650 \mathrm{~cm}^{-1}$ (amide I band), 


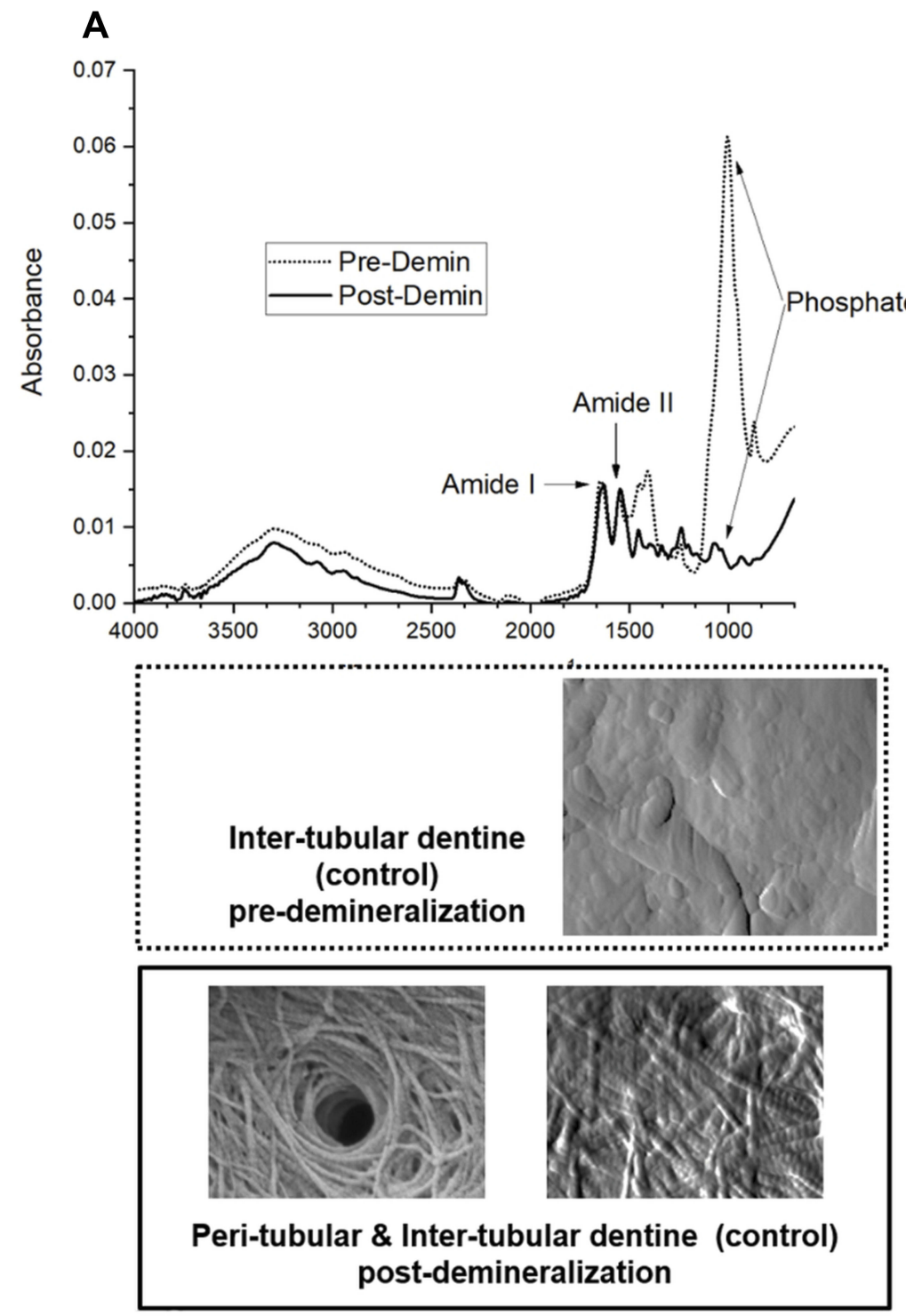

B
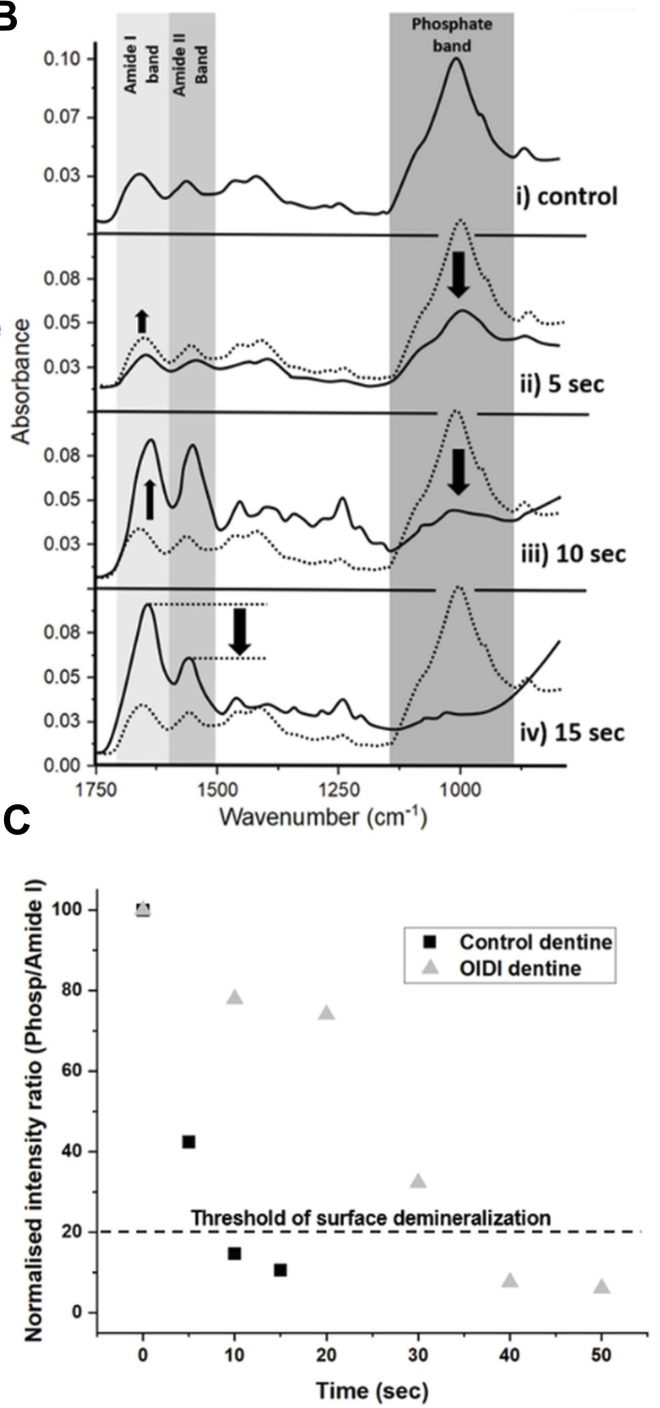

Figure 2 FTIR spectra (A) averaged composite FTIR spectra of control teeth pre-demineralisation (dotted line) and post-demineralisation (solid line). Images (SEM and AFM) present the control dentin surface pre- and post-demineralisation. (B) Infrared spectrum of the control (i) dentine, after $5 \mathrm{~s}$ (ii), $10 \mathrm{~s}$ (iii) and I5 $\mathrm{s}$ (iv) demineralisation. (C) Plot of pf the normalized intensity band ratio (Phosphate/Amide I) as function of the demineralisation time for control and OIDI dentin; dotted line marks the empirical threshold below which the surface is considered fully demineralised.

the $\mathrm{N}-\mathrm{H}$ and $\mathrm{C}-\mathrm{N}$ bonds at $1550 \mathrm{~cm}^{-1}$ (amide II band) and at $1230 \mathrm{~cm}^{-1}$ (amide III band). By comparing the relative absorbance intensity of the $\mathrm{PO}_{4}{ }^{3}$ band relatively to that of the Amide I and II, it is possible to assess the level of demineralization in the dentin following an acid exposure.

Figure 2B shows a series of ATR-FTIR spectra (notnormalized) recorded on the same control dentin section exposed to an increased exposure of phosphoric acid from $0 \mathrm{~s}$ up to $15 \mathrm{~s}$. In this figure, one can appreciate the constant decrease of the $\mathrm{PO}_{4}{ }^{3}$ band already after 5 $\mathrm{s}$ exposure to phosphoric acid. After $10 \mathrm{~s}$, this band has almost completely disappeared whereas the collagen
Amide I and II doublet become more and more apparent. If the exposure to phosphoric acid is increased up to $15 \mathrm{~s}$, the intensity of the Amide II starts decreasing suggesting that the acid may be starting to degrade the collagen itself. The gradual decrease in intensity of the $\mathrm{PO}_{4}{ }^{3}$ band confirms that the mineral phase present within the first $2 \mu \mathrm{m}$ from the surface of the dentin section is being removed by acid exposure. One of the reasons for choosing ATR-FTIR over other spectroscopic techniques, such as Raman for example, is that the depth of sampling in ATR is governed by the evanescent infrared waves emerging from the diamond window. The depth of penetration of these waves in 
the sample is only a few microns $(<2 \mu \mathrm{m})$ moderated by the refractive indices of both the sample and diamond window. Thus, our approach ensures that we are only sampling a surface demineralization rather than a full thickness demineralization. Finally, it is difficult to assess the impact of this protocol onto the phosphorylated noncollagenous proteins as their individual fingerprints have not yet been established using ATR-FTIR spectroscopy. These are likely to combine both the Amide I and the $\mathrm{PO}_{4}{ }^{3-}$ bands but further studies are required to prove this.

By calculating the normalised ratio between the intensities of the phosphate band over the Amide I band as a function of acid-exposure time, one can appreciate the increase in the exposure time ( $10 \mathrm{~s}$ for control and $35 \mathrm{~s}$ for OIDI) required to observe a net reduction of the phosphate band presence in the infrared spectra. To date, there have been no reports on the effects of mineralization of OIDI on dentin which may explain for the longer required demineralisation time. However, studies have shown increased mineralization to be a characteristic feature of OI bone achieved by densely packed mineral particles as a result of defective collagen, leading to high fragility and a brittle glass-like nature. ${ }^{21-23}$ As a result, we empirically defined a threshold value (20\%) for the calculated ratio of the two fingerprint bands to mark when surface demineralisation has been achieved when monitored by ATR-FTIR. The demineralisation of all the sections (control and OIDI) were carried up to the point where the normalised ratio of the two fingerprints (Phosphate and Amide I) fell below this threshold value. This ensured that our systematic demineralization of the control and OIDI dentin surface became reliable even when using $37 \%$ phosphoric acid as shown in Figure 2C.

\section{Morphological Assessment of Demineralized Dentin Surface}

Although the clinical presentation of OIDI varies from patient to patient, OIDI-affected primary teeth will often have short roots and bulbous crowns, with a significant constriction at the cement-enamel junction (CEJ) and pulpal obliteration. The primary dentition is normally more severely affected than the permanent teeth. ${ }^{24}$ Although these teeth also present an increased susceptibility to wear as a result of lower enamel hardness and resistance to attrition, it is understood that structural changes in the dentin may explain the failure of some adhesive restorative materials. ${ }^{8}$ However, there is currently no reports detailing such structural changes. In dentin, it is the composite nature of the mineralised collagen scaffold that provides the tooth with its mechanical integrity. Forming an ordered structure, the collagen fibrils present in dentin are arrayed in a consistent distribution of interrupted striations and thinner fibrils, thus forming an overall collagen network. The remaining non-collagenous part of dentin is composed of non-structural proteins. Any alteration of this ordered array at the mesoscale, or fibrillar ultrastructure at the submicroscale, may result in an increased susceptibility to fracture as reported elsewhere. ${ }^{8}$

\section{Mesoscale Ultrastructure of Demineralised Dentin}

Figure 3 shows representative images of both the demineralised control dentin a) and demineralised OIDI dentin at various magnifications. In the case of the control sample, one can observe the regular distribution of dentinal tubules across the surface of the samples (at the lowest magnification image 2-a-i). These tubules are also all unblocked as expected following the $\mathrm{NaOCl}$ step in our protocol. Imaging the demineralized control dentin sample at higher magnification, it is possible to observe the exposed collagen matrix on the surface of the sample, both around and inside the tubules as presented in Figure 3A-ii, iii and iv. In these images, clear interstitial gaps between the fibrils can be observed suggesting that the demineralization protocol used was effective in removing the mineral matrix that surrounds the collagen matrix. At the higher magnification (2-a-iii \& iv), it is possible to evaluate whether the demineralization protocol may have affected the topology of the collagen fibrils. As one can clearly identify the collagen D-banding periodicity without any signs of fibrils unwinding or swelling, it is likely that our controlled demineralisation has not affected the collagen fibrils morphology.

In the case of the OIDI, as presented in Figure 3B-i, very few tubules are present at the surface of the sample. Those present are more ovoid in shape which could be due to the angle at which the sections were cut. Figure 3B-ii shows a "nesting" effect of tubules, which can only be observed in OIDI samples. This nesting or branching of the tubules was also observed histologically by Andersson et al. ${ }^{25}$ In their study, they also reported variations in the width of the dentin tubules, presence of hyaline dentin void of dentin tubules, layering of dentin, and presence of cell lacunae and duct-like structures in the body of the dentin. This corroborated with our findings, including the diameter of tubules which appears to be also less uniform in our images. We 

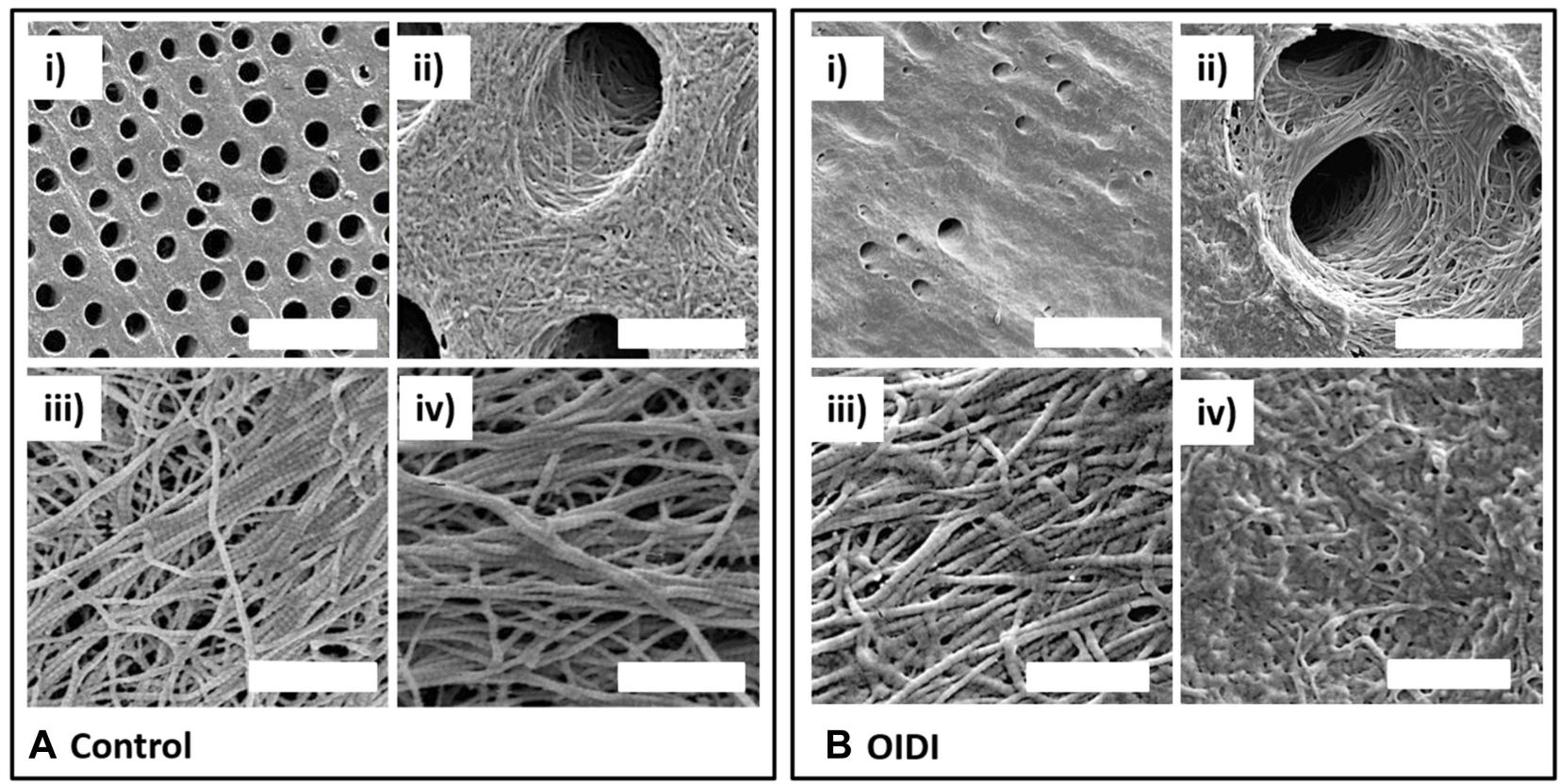

Figure 3 (A) SEM images of control dentin: i) pre-demineralisation (scale bar $20 \mu \mathrm{m}$ ): regular dentinal tubule arrangement, tubules are open and uniform in appearance; ii) post demineralisation (scale bar $2 \mu \mathrm{m}$ ): higher magnification image of tubules, collagen scaffold is visible inside the tubules and in the surrounding space; iii) and iv) post demineralisation (scale bar $800 \mathrm{~nm}$ ): higher magnification images showing regular D-banding periodicity of collagen fibrils. (B) SEM images of OIDI dentin: i) predemineralisation (scale bar $20 \mu \mathrm{m}$ ): tubules varying in size and partially blocked or occluded; ii) post demineralisation (scale bar $2 \mu \mathrm{m}$ ): "nesting" of several tubules can be seen within a larger tubule, collagen can be seen in the tubule (scale bar $2 \mu \mathrm{m}$ ), iii) post demineralisation (scale bar $800 \mathrm{~nm}$ ): higher magnification image showing regular D-banding periodicity of collagen fibril; iv) post demineralisation (scale bar $800 \mathrm{~nm}$ ): higher magnification image showing collagen fibril coalescence.

should, however, be careful with this outcome as tooth sectioning angle may affect the cross-sectional uniformity of the dentine tubules. Although the OIDI samples were subjected to the same sonication protocol as used for the control samples, partial dentinal tubules occlusions can be also observed in Figure 2B-i. We suggest that a tubule is partially occluded when we can observe its outline, but its void is filled with non-specific compounds. At this stage, it is difficult to evaluate if the presence of these partial occlusions is phenotypically relevant to OIDI as this finding has not yet been reported elsewhere to date.

Contrary to the control samples, two types of morphological collagen scaffold organisation could be observed at higher magnification. Figure 3B-iii presents a very uniform collagen scaffold very reminiscent to that of the control dentin. The D-banding periodicity is very apparent on all collagen fibrils with no obvious sign of structural damage. However, in Figure 3B-iv, the collagen network has been significantly altered and the fibrils network is no longer homogeneous. In fact, the topology of the fibrils suggests a significant level of intro-fibrillar disorganising with notable fibrillar diameter swelling and constriction. Sporadic presence of D-banding periodicity can be observed on a few sparse fibrils, but overall the fibrils coalesce together amorphously. The prevalence of such morphologically compromised area over the entire OIDI dentin sections was not quantified and therefore should be carefully considered.

\section{Quantitative Assessment of Dentin Tubules}

For the control dentin, a total of 1104 tubules were identified over 175 regions $\left(20 \times 20 \mathrm{um}^{2}\right.$ each), averaging 6.3 tubules per area. For the OIDI dentin, a total of 223 tubules were identified over 137 regions $\left(20 \times 20 \mu \mathrm{m}^{2}\right.$ each), averaging 1.6 tubules per area. Compared to the OIDI sample, the native control samples presented on average over 5 times more tubules per area investigated although the measurements were performed at the same locations on all teeth. Here we can define dentine density at a ratio between dentine (as a continuous bulk matrix) and the number of tubules present per unit area. This clear difference in the tubules number per unit area suggests that the OIDI dentin is much denser than the control dentin. This could be linked to either a diminished odontoblastic activity or increased mineralization towards tubule occlusion in OIDI patients. From a mechanical point of view, this apparent density should provide the OIDI dentin with a greater resistance to fracture. A significant difference in tubule diameter between the 2 groups could also be recorded as presented in Figure 4A. In the case of the native control 


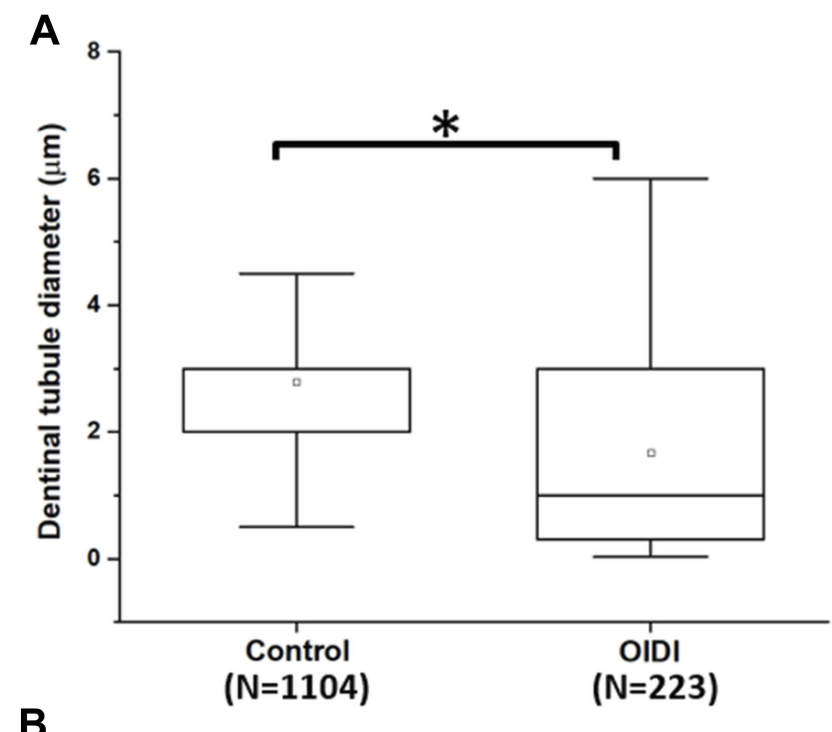

B
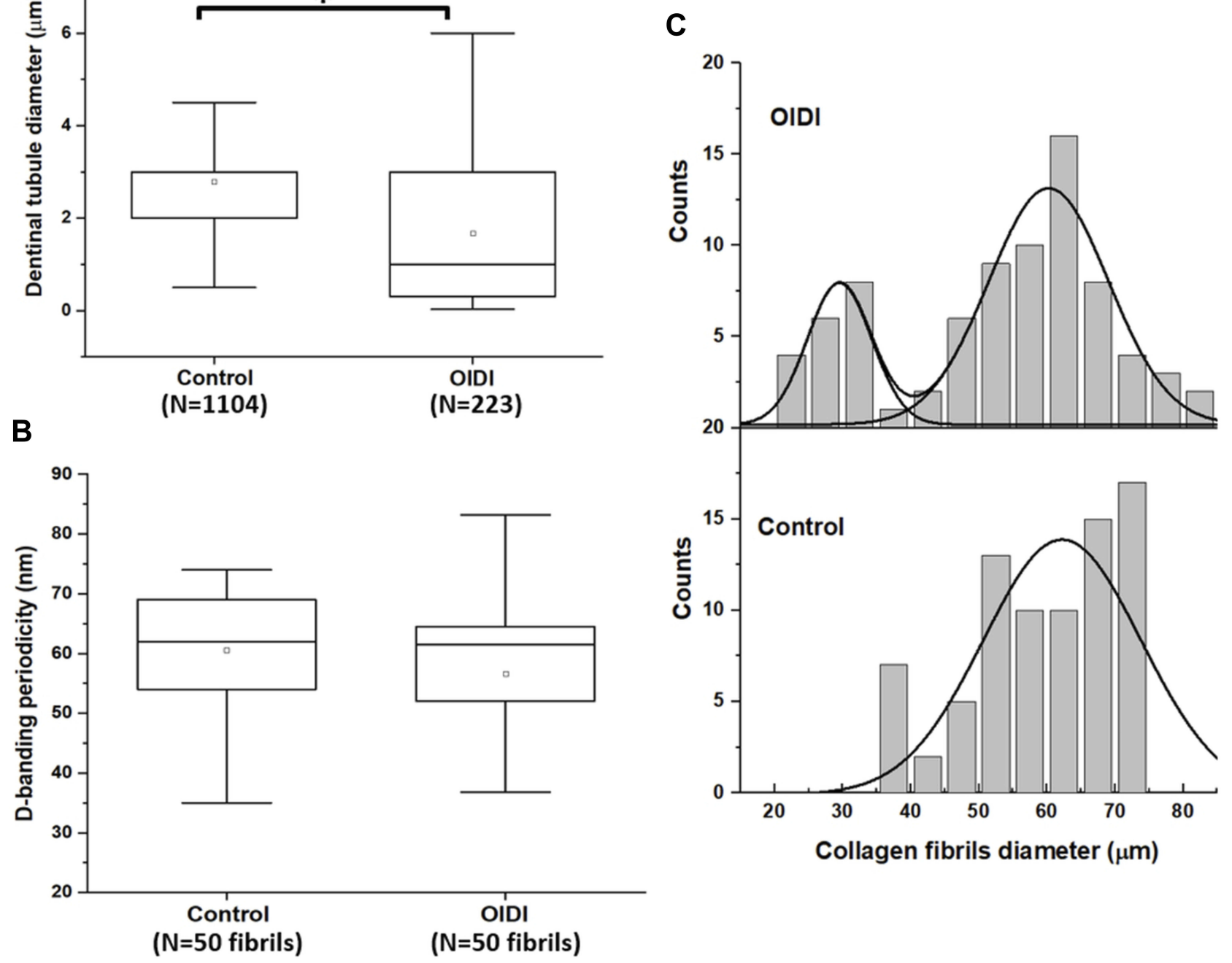

Figure 4 Graphical presentation of the nanoscale phenotypic properties (structural) for control and OIDI dentin: (A) boxplots of the measured dentinal tubules diameter (*significance $\mathrm{p}<0.05$ ). (B) Boxplots of the measured collagen fibrils D-banding periodicity (no significant differences were observed). (C) Fitted histograms of the collagen fibril diameter with bimodal distributions (Gaussian) for OIDI collagen $\left(R^{2}>0.9\right)$ and for control collagen $\left(R^{2}>0.8\right)$.

sample, the tubule diameter was found to be narrowly distributed, $2.75 \pm 0.72 \mu \mathrm{m}$ in diameter, whereas in the case of the OIDI samples, not only does the median diameter decrease to $1.67 \pm 0.11 \mu \mathrm{m}$ but the distribution of the diameter is also increased moderately when compared to that of native control dentin. Statistical analysis using Kruskal-Wallis ANOVA shows a significant difference $(\mathrm{P}<0.05)$ between the tubule diameters in OIDI and control, with the control tubules being larger. Since the formation of tubules is directly influenced by both the odontoblasts as to form the collagen matrix and the odontoblast processes around which the dentine matrix mineralizes, it is plausible to hypothesize that the variation in dentinal tubules diameter could be directly linked an alteration to the collagen scaffold deposited. This is yet to be investigated as much needs to be done to elucidate the influence between odontoblastic activity and OIDI prevalence.

\section{Quantitative Assessment of Collagen Fibrils Structure}

The ultrastructure of the exposed matrices in both native control and OIDI demineralized dentin was also investigated by using higher magnification electron microscopy and AFM imaging as presented in Figure 3A-iii and B-iii. In both control and OIDI images, collagen fibrils can be clearly observed presenting a well-defined D-banding periodicity, in a regular, ordered state. D-banding periodicity 
is an intrinsic topographical feature of native collagen fibrils. $^{26-28}$ Together with an overall regular fibrillar topography, this D-banding can be used as a marker for intact collagen as both the shape of the fibrils, and the width of the D-banding. The lack of D-banding may be the result of an internal structural and molecular disorganisation, enzyme-mediated digestion, acid or thermal denaturation. Using these images, it was possible to measure the distribution of D-banding and collagen fibril diameter in both the series of samples. As shown in Figure 4B, both native control and OIDI presented a unimodal distribution with medians of $(63.2 \pm 0.2) \mathrm{nm}(\mathrm{N}=50$ fibrils $)$ and $(62.3 \pm 0.3)$ $\mathrm{nm}$ ( $\mathrm{N}=50$ fibrils), respectively. Kruskal-Wallis testing confirmed that there were no significant differences in $\mathrm{D}$-banding length $(\mathrm{P}>0.05)$. The spread of $\mathrm{D}$-banding value was however larger in the case of OIDI spanning from 50 to $85 \mathrm{~nm}$, whereas this value was 52 to $75 \mathrm{~nm}$ in the control samples. The average D-banding length found for both the native control and OIDI was also comparable to the commonly reported average of $67 \mathrm{~nm} .^{29-31}$ This average however often refers to the D-banding periodicity length found in collagen in skin or tendon, and very little data have been reported on teeth until now.

Interestingly, the distribution of the fibril diameters in this study presented clear differences between the study groups. The native control sample fibrils' diameter was uniformly distributed over the range 50 to $75 \mathrm{~nm}$ where the OIDI fibrils diameter was bi-modally distributed. The large population of fibrils diameter were found to have a median of $62.1 \mathrm{~nm}$, whereas the smaller population of fibrils had a median of $30.7 \mathrm{~nm}$ as presented in Figure 4C. This type of bimodal distribution is more reminiscent of tendons than bones or dentin. $^{31,32}$ The population of fibrils with the lower diameter is often associated with immature fibrils on tendons. Following this possible reasoning, our results may suggest that there is significant proportion of immature collagen fibrils present in OIDI dentin, which may result in weaker mechanical properties for the dentin structure and the tooth overall. Unlike tendons or bones, there is no direct regeneration through the combined osteoclasts/osteoblast activities in the dentin. Dentin is a calcified tissue formed through dentinogenesis, which results in the conversion of un-mineralized predentin into mineralized dentin. ${ }^{33}$ As odontoblast cells move towards the pulp, they form an odontoblastic process, which penetrates the un-calcified predentin shaping the dentinal tubules. ${ }^{34}$ Thus, once the odontoblast cells have remodelled the dentin, it is accepted that the dentin and its constituent remain unaltered throughout the lifetime of the tooth, although these can be subjected to exogeneous factors (caries, for example). The presence of this bi-modal fibril diameter population is therefore not a result of fibril growth during the lifetime of the tooth but is already present at the dentinogenesis stage. It is plausible to consider that this variation in collagen fibrils diameter could be one of the key factors affecting the overall dentin mechanical stability and entire tooth prognosis in the case of OIDI.

\section{Mechanical Properties of Dentin vs Dentinal Collagen \\ Dentin Hardness}

To evaluate the bulk mechanical properties of OIDI dentin versus control dentin, hardness measurements were performed directly on the sections prior to demineralisation. The hardness of a tissue determines its resistance to deformation, scratching, or abrasion. Additionally, hardness is described as the capability to resist permanent indentation. ${ }^{35}$ In the case of teeth, it is a valid indicator to assess the tooth or dentin resistance to fracture. As presented in Figure 5A, the hardness of the control dentin was found to be significantly higher $(p<0.05)$ than that of the OIDI. The mean hardness of the control dentin was found to be $\mathrm{VHN}_{\text {control }}=56.4 \pm 3.7(\mathrm{~N}=80$ indentations) while the mean hardness of the OIDI dentin was found to be $\mathrm{VHN}_{\mathrm{OIDI}}=21.9 .4 \pm 2.0$ ( $\mathrm{N}=80$ indentations). The hardness of the OIDI is less than half of that of control dentin. This result confirms the expected result of this study that OIDI dentin is more fragile than control dentin as suggested by another study. ${ }^{36}$ When considering the hardness of dentin, it is particularly important to evaluate its mineral contents. Kinney et $\mathrm{al}^{37}$ measured the mineral content in DI type IIaffected and normal dentin using high-resolution synchrotron radiation computed tomography and found that the mineral concentration was $33 \%$ lower on average in the DI type II dentin with respect to normal dentin. Unlike the form of dentinogenesis (OIDI) that is linked with osteogenesis imperfecta, DI type II is not believed to be associated with any known defects in collagen structure. However, as OIDI is caused by abnormal type I collagen synthesis, it is highly likely that the interaction between collagen and minerals may also be affected and as a result, this would lead to poor mechanical properties.

\section{Collagen Fibril Mechanical Properties}

To evaluate if OIDI had any mechanical impact on dentinal collagen at the single fibril level, it was essential to ensure that only collagen fibrils with intact morphology would be characterized. As such, we omitted any area 

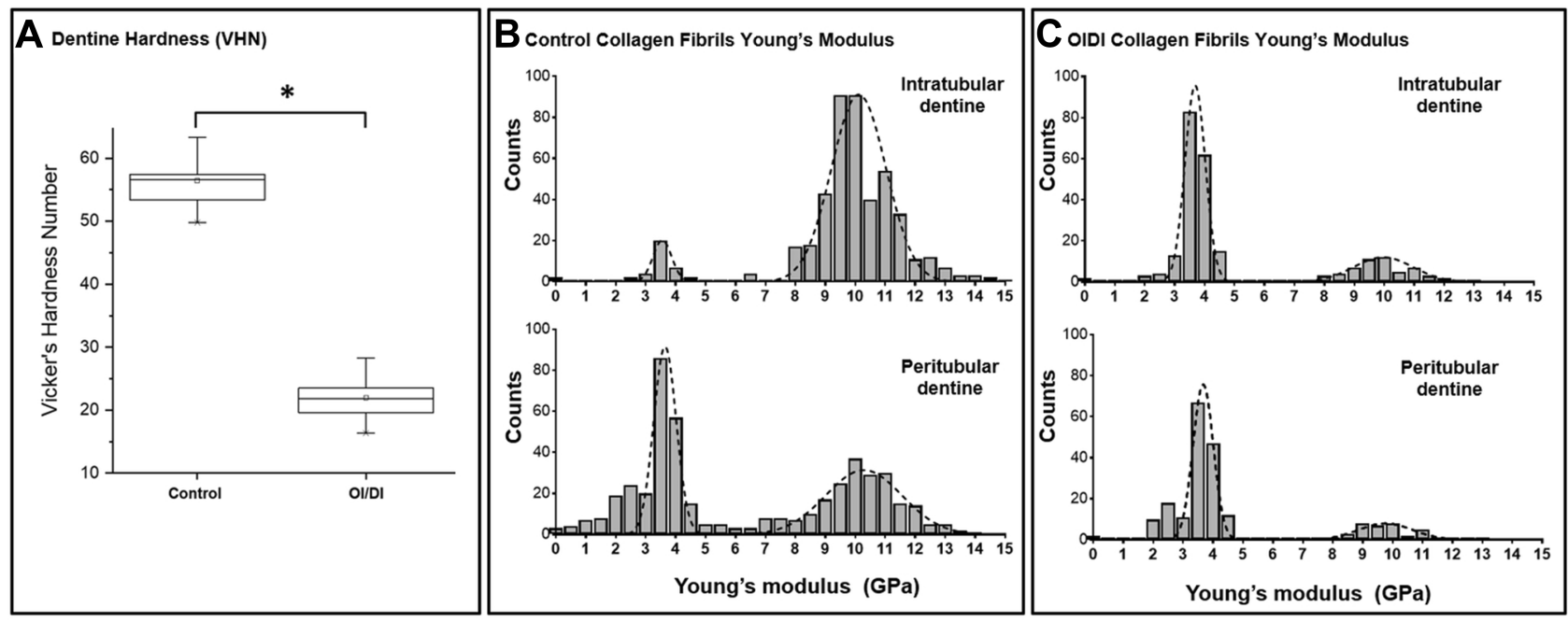

Figure 5 Graphical presentation of the nanoscale phenotypic properties (mechanical) for control and OIDI dentin (A) boxplots of the Vickers hardness value (*significance $\mathrm{p}<0.05$ ). (B and $\mathbf{C}$ ) Fitted histograms of the collagen fibril Young's modulus with bimodal distributions (Gaussian $R^{2}>0.9$ for all distributions) as a function of measurement site: intratubular and peritubular, respectively.

previously described as weakened from this mechanical analysis as it was not possible to assert whether the topological changes where due to the demineralization protocol or if these are inherently associated with the disease. Thus, the AFM was used in imaging mode to identify collagen present with clear D-banding periodicity. By performing a systematic mechanical analysis, ${ }^{38}$ it was then possible to extract mechanical properties of individual collagen fibrils immediately surrounding the tubules (denoted intratubular) and equidistantly between tubules (denoted peritubular). Figure $5 \mathrm{~B}$ and $\mathrm{C}$ shows the profile for the elastic (Young's) modulus of the control and OIDI collagen fibrils, respectively. In Figure 5B, the values of collagen elastic modulus for the control intratubular and peritubular regions followed a reverse mechanical profile. In the case of control intratubular dentin, the bimodal distribution with the greater population is centred at $10.4 \mathrm{GPa}$ whereas a smaller population is centred at $3.6 \mathrm{GPa}$. In the case of control peritubular dentin, the bimodal distribution with the greater population is centred at $3.8 \mathrm{GPa}$ whereas a smaller population is centred at $10.3 \mathrm{GPa}$. From the process of dentinogenesis, it is known that odontoblasts lay down the primary dentin in the peritubular region first, before reinforcing and withdrawing through the tubules. The reinforcement method of collagen is by nanoscale chemical cross-linking, and previous research from our group has shown that these changes can be detected. ${ }^{12}$ The data of the control supports this; the peritubular collagen has not been crosslinked to the extent that the intertubular collagen has, and so it is weaker.
In Figure 5C, the values of collagen elastic moduli for the OIDI intratubular and peritubular regions do not follow the same pattern as observed for the control sample. In fact, the collagen fibrils from both the intra and peritubular dentin present the same bimodal mechanical distribution. The largest population of collagen elastic moduli values for both the regions is centred around 3.8GPa whereas the smallest population for the collagen elastic moduli values is centred around 10.0 GPa and 9.8 GPa for the intratubular and the peritubular regions, respectively. This lack of reverse pattern in the collagen elastic moduli in the case of OIDI with respect to the distance from the tubule is indicative that the collagen is mechanically more uniform throughout the dentin thickness, albeit being also mechanically weak.

The formation of dentinal tubules is the direct outcome of the migration of differentiated odontoblasts towards the dental papilla, leaving behind processes around which the dentine matrix mineralizes. Yet, histologically, and microscopically, these tubules appear as tubular voids within the dense mineral matrix that is dentine. For a structural and mechanical point of view, the presence of these tubular voids ought to weaken the composite material properties of dentine. Yet, collagen fibrils immediately surrounding the tubules were found to be mechanically stiffer in the control dentin when compared to the fibrils present equidistantly between dentine tubules. This ought to suggest that the collagen fibrils immediately surrounding the tubules are mechanically reinforced to support the interruption dentine structure created by the presence of tubular 
voids (dentine tubules). However, the OIDI samples did not show this region-dependent change in stiffness. This would suggest that the dentine structure is not reinforced around the dentinal tubules, which would lead to overall weaker dentine mechanical and structural properties. A plausible reason for this to occur may be linked to the inability of the collagen present in these intra-tubular regions, the case of OIDI to respond to extra-cellular posttranslational processing such as crosslinking for example.

Although more research would be required to evaluate the crosslinking profiles of regions specific collagen, this finding suggests that the quality of the collagen fibrils is mechanically poorer in the case of dentinogenesis imperfecta associated with osteogenesis imperfecta, which in turn reduce the dentine structural and mechanical properties.

\section{Clinical Impact}

Dentinal collagen plays an important role in the stability of dentin but unlike in bones, it is not renewed during the lifetime of the tooth. Thus, any endogenous (genetic, disease) or exogenous alteration ${ }^{21}$ to the collagen network will impact both its structure and function as part of a functional mineralised tissue. In the case of OIDIaffected teeth, we have demonstrated in an exploratory approach how dentinal collagen fibrils exhibit a bimodal distribution in terms of diameter which is not found in control samples. Correlating this finding with the observed lack of increased elastic moduli of the collagen fibrils in the intratubular OIDI dentin may be a first explanation towards the definition of structural changes in the dentin responsible for the failure of some adhesive restorative materials as observed in patients affected by OIDI.

Mild types of OI can be difficult to diagnosis clinically, therefore dental assessment, including clinical and radiographic examination, can be important to aid diagnosis. ${ }^{39}$ Children with suspected OI should always be screened by a specialist paediatric dentist, as dental management of OIDI children can be complex. Whilst preformed metal crowns are suitable for restoration of primary molars, due to the increased tendency of composite restorations to fail in DI patients; glass ionomer materials are commonly used as means of restoring anterior teeth as they do not depend on mechanical interlocking with collagen. ${ }^{40}$ It is unclear whether the mode of restoration and improper etching of teeth prior to applying dental composites, or possible dentinal matrix or collagen alterations, that might be ultra-structurally defective and mechanically weaker thus forming an ineffective hybrid layer during bonding, which can be a causative factor in failure. ${ }^{40}$

\section{Conclusion}

This study presents a systematic approach to the characterization of collagen structure and properties in OIDI as diagnosed in dentin. A modified demineralization protocol comprising acid sonication resulted in much clearer topographic images of exposed dentinal collagen. In our approach, we propose that demineralization protocols should ideally be sample-specific and tailored to each sample examined. After the analysis of data obtained from AFM and SEM imaging, it was found that to study the collagen ultrastructure within a mineralized matrix, it was essential to reach a balance between mineral removal and collagen exposure, whilst avoiding the denaturation of the collagen matrix itself. Structural markers for OIDI at the mesoscale and nanoscale were found and supported by a mechanical analysis. D-banding periodicity average values were consistent with the literature, but were statistically insignificant when compared with OIDI samples. However, dentinal tubule number and collagen fibril diameter, and the overall fibril meshwork arrangement, were seen to vary considerably. One of the main outcomes of this study was to explore the topography of the collagen network and its mechanical properties in the case of OIDI. New questions and challenges have been raised regarding the quality of the collagen network in the case of DI associated with OI, its impact upon treatment strategies in dentistry, and ultimately the correct treatment protocol to most benefit patients.

\section{Acknowledgements}

The authors would like to thank First 20 Foundation, Egypt, for sponsoring the main body of work and a scholarship for Dr S. Ibrahim. Acknowledgement of scholarship support for this study from Ministry of Higher Education Malaysia and the Islamic Science University of Malaysia for the scholarship (Dr N. Mohamed Ibrahim \& Dr N. Harith) \& Ministry of Defence, Saudi Arabia (Dr A. Shinawi). The preliminary findings of this paper were presented as a conference talk at the Internal Association of Dental Research (IADR) 2017 at San Francisco.

\section{Author Contributions}

All authors contributed to data analysis, drafting or revising the article, gave final approval of the version to be published, and agree to be accountable for all aspects of the work. 


\section{Disclosure}

The authors report no conflicts of interest in this work.

\section{References}

1. Shields ED, Bixler D, El-Kafrawy AM. A proposed classification for heritable human dentin defects with a description of a new entity. Arch Oral Biol. 1973;18:543-553. doi:10.1016/0003-9969(73)90075-7

2. Witkop CJ, Jr. Hereditary defects of dentin. Dent Clin North Am. 1975;19:25-45.

3. Devaraju D, Devi BY, Vasudevan V, Manjunath V. Dentinogenesis imperfecta type I: a case report with literature review on nomenclature system. J Oral Maxillofac Pathol. 2014;18(Suppl 1). doi:10.4103/0973-029X.141363

4. Andersson K, Malmgren B, Astrom E, Dahllof G. Dentinogenesis imperfecta type II in Swedish children and adolescents. Orphanet $J$ Rare Dis. 2018;13:145. doi:10.1186/s13023-018-0887-2

5. Kim JW, Simmer JP. Hereditary dentin defects dentin. J Dent Res. 2007;86(5):392-398. doi:10.1177/154405910708600502

6. American Academy of Pediatric Dentistry. Guideline on dental management of heritable dental developmental anomalies. Pediatr Dent. 2013;35(5):E179-E184.

7. Pettiette MT, Wright JT, Trope M. Dentinogenesis imperfecta: endodontic implications. Oral Surg Oral Med Oral Pathol Oral Radiol Endodontol. 1998;86:733-737. doi:10.1016/S1079-2104(98)90213-X

8. Wieczorek A, Loster J. Dentinogenesis imperfecta type II: ultrastructure of teeth in sagittal sections. Folia Histochem Cytobiol. 2013;51 (3):244-247. doi:10.5603/FHC.2013.0035

9. Barron MJ, McDonnell ST, Mackie I, Dixon MJ. Hereditary dentin disorders: dentinogenesis imperfecta and dentin dysplasia. Orphanet $J$ Rare Dis. 2008;3:31. doi:10.1186/1750-1172-3-31

10. Tsai C-L, Lin Y-T, Lin Y-T. Dentinogenesis imperfecta associated with osteogenesis imperfecta: report of two cases. Chang Gung Med J. 2003;26:138-143.

11. Thotakura SR, Mah T, Srinivasan R, Takagi Y, Veis A, George A. The non-collagenous dentin matrix proteins are involved in dentinogenesis imperfecta type II (DGI-II). Res Reports Biol. 2000;79(3):835-839.

12. Herchenhan A, Uhlenbrock F, Eliasson P, et al. Lysyl oxidase activity is required for ordered collagen fibrillogenesis by tendon cells. $J$ Biol Chem. 2015;290(26):16440-16450. doi:10.1074/jbc.M115.641670

13. Nuytinck L, Wettinck K, Freund M, et al. Osteogenesis imperfecta phenotypes resulting from serine for glycine substitutions in the $\alpha 2$ (I) collagen chain. Eur $J$ Human Genet. 1997;5(3):161-167. doi: $10.1159 / 000484752$

14. Stöss H, Freisinger P. Collagen fibrils of osteoid in osteogenesis imperfecta: morphometrical analysis of the fibril diameter. $\mathrm{Am}$ J Med Genet. 1993;45(2):257. doi:10.1002/(ISSN)1096-8628

15. Marini JC, Lewis MB, Wang Q, Chen KJ, Orrison BM. Serine for glycine substitutions in type I collagen in two cases of type IV osteogenesis irnperfecta (OI). J Biol Chem. 1993;268(4):2667-2673.

16. Costa LRRS, Watanabe IS, Kronka MC, Silva MCP. Structure and microstructure of coronary dentin in non-erupted human deciduous incisor teeth. Braz Dent J. 2002;13(3):170-174. doi:10.1590/S010364402002000300005

17. Ben Amor IM, Glorieux FH, Rauch F. Genotype-phenotype correlations in autosomal dominant osteogenesis imperfecta. $J$ Osteoporos. 2011;2011:540178. doi: 10.4061/2011/540178

18. Rauch F, Lalic L, Roughley P, Glorieux FH. Relationship between genotype and skeletal phenotype in children and adolescents with osteogenesis imperfecta. J Bone Miner Res. 2009;25(6):1367-1374. doi:10.1359/jbmr.091109

19. Rauch F, Lalic L, Roughley P, Glorieux FH, Genotype-phenotype correlations in nonlethal osteogenesis imperfecta caused by mutations in the helical domain of collagen type I. Eur J Hum Genet. 2010;18 (6):642-647. doi:10.1038/ejhg.2009.242
20. Chyasnavichyus M, Young SL, Geryak R, Tsukruk VV. Probing elastic properties of soft materials with AFM: data analysis for different tip geometries. Polymer. 2016;102:317-325. doi:10.1016/j. polymer.2016.02.020

21. Redha O, Strange A, Maeva A, et al. Impact of carbamide peroxide whitening agent on dentinal collagen. $J$ Dent Res. 2019;98 (4):443-449. doi:10.1177/0022034518822826

22. Boyde A, Travers R, Glorieux FH, Jones SJ. The mineralization density of iliac crest bone from children with osteogenesis imperfecta. Calcif Tissue Int. 1999;64(3):185-190. doi:10.1007/ s002239900600

23. Fratzl-Zelman N, Misof BM, Klaushofer K, Roschger P. Bone mass and mineralization in osteogenesis imperfecta. Wiener Medizinische Wochenschrift. 2015;165(13-14):271-277. doi:10.1007/s10354-0150369-2

24. Jones SJ, Glorieux FH, Travers R, Boyde A. The microscopic structure of bone in normal children and patients with osteogenesis imperfecta: a survey using backscattered electron imaging. Calcif Tissue Int. 1999;64(1):8-17. doi:10.1007/s002239900571

25. Kristofer Andersson K, Dahllöf G, Lindahl K, et al. Mutations in COL1A1 and COL1A2 and dental aberrations in children and adolescents with osteogenesis imperfecta - a retrospective cohort study. PLoS One. 2017;12:5.

26. Majorana A, Bardellini E, Brunelli P, Lacaita M, Cazzolla A, Favia G. Dentinogenesis imperfecta in children with osteogenesis imperfecta: a clinical and ultrastructural study. Int J Paediatr Dent. 2010;20(2):112-118. doi:10.1111/j.1365-263X.2010.01033.x

27. Lingham-Soliar T, Wesley-Smith J. First investigation of the collagen D-band ultrastructure in fossilized vertebrate integument. Proc Biol Sci. 2008;275(1648):2207-2212. doi:10.1098/rspb.2008.0489

28. Ortolani F, Giordano M, Marchini M. A model for type II collagen fibrils: distinctive D-band patterns in native and reconstituted fibrils compared with sequence data for helix and telopeptide domains. Biopolymers. 2000;54(6):448-463. doi:10.1002/(ISSN)1097-0282

29. Yang B, Adelung R, Ludwig K, Boessmann K, Pashley DH, Kern M. Effect of structural change of collagen fibrils on the durability of dentin bonding. Biomaterials. 2005;26(24):5021-5031. doi:10.1016/ j.biomaterials.2005.01.024

30. Strange AP, Aguayo S, Ahmed T, et al. Quantitative nanohistological investigation of scleroderma: an atomic force microscopy-based approach to disease characterization. Int $J$ Nanomedicine. 2017;12:411-420. doi:10.2147/IJN

31. Holmes DF, Gilpin CJ, Baldock C, Ziese U, Koster AJ, Kadler KE. Corneal collagen fibril structure in three dimensions: structural insights into fibril assembly, mechanical properties, and tissue organization. Proc Natl Acad Sci USA. 2001;98(13):7307-7312. doi:10.1073/pnas. 111150598

32. Fang M, Holl MMB. Variation in type I collagen fibril nanomorphology: the significance and origin. Bonekey Rep. 2013;2:394. doi:10.1038/bonekey.2013.128

33. Goh KL, Holmes DF, Lu Y, et al. Bimodal collagen fibril diameter distributions direct age-related variations in tendon resilience and resistance to rupture. $J$ Appl Physiol. 2012;113(6):878-888. doi:10.1152/japplphysiol.00258.2012

34. Youravong N, Teanpaisan R, Norén JG, et al. Chemical composition of enamel and dentin in primary teeth in children from Thailand exposed to lead. Sci Total Environ. 2008;389(2-3):253-258. doi:10.1016/j.scitotenv.2007.08.053

35. Niño-Barrera JL, Gutiérrez ML, Garzón-Alvarado DA. A theoretical model of dentinogenesis: dentin and dentinal tubule formation. Comput Methods Programs Biomed. 2013;112(1):219-227. doi:10.1016/j.cmpb.2013.06.010

36. Min B, Song JS, Lee JH, et al. Multiple teeth fractures in dentinogenesis imperfecta: a case report. J Clin Pediatr Dent. 2014;38 (4):362-365. doi:10.17796/jcpd.38.4.q523456j733642r2 
37. Kinney JH, People JA, Driessen CH, et al. Intrafibrillar mineral may be absent in dentinogenesis imperfecta type II (DI-II). J Dent Res. 2001;80(6):1555-1559. doi:10.1177/00220345010800061501

38. Wenger MPE, Bozec L, Horton MA, Mesquida P. Mechanical properties of collagen fibrils. Biophys J. 2007;93(4):1255-1263. doi:10.1529 biophysj.106.103192
39. Malmgren B, Norgren S. Dental aberrations in children and adolescents with osteogenesis imperfecta. Acta Odontol Scand. 2002;60 (2):65-71. doi:10.1080/000163502753509446

40. Perdigão J, Monteiro P, Gomes G. In vitro enamel sealing of self-etch adhesives. Quintessence Int (Berl). 2009;40(3):225-233.

\section{Publish your work in this journal}

The International Journal of Nanomedicine is an international, peerreviewed journal focusing on the application of nanotechnology in diagnostics, therapeutics, and drug delivery systems throughout the biomedical field. This journal is indexed on PubMed Central, MedLine, CAS, SciSearch ${ }^{\mathbb{B}}$, Current Contents ${ }^{\mathbb{B}} /$ Clinical Medicine, $^{2}$
Journal Citation Reports/Science Edition, EMBase, Scopus and the Elsevier Bibliographic databases. The manuscript management system is completely online and includes a very quick and fair peer-review system, which is all easy to use. Visit http://www.dovepress.com/ testimonials.php to read real quotes from published authors. 